\title{
Accelerator Mass Spectrometry: Ultra-sensitive Detection Technique of Long-lived Radionuclides
}

\author{
Hans-Arno Synal*
}

\begin{abstract}
An introduction is given to accelerator mass spectrometry (AMS) technology, to the fundamental measurement principles, and the physics aspects behind the design constraints of AMS instruments. This article shall give an overview on technical design constrains of AMS instrumentation, general ion optical principles, and nuclide specific problems. The historic development of AMS detection techniques is briefly summarized. The wide variety of applications connected to the AMS technology are not discussed.
\end{abstract}

Keywords: AMS · Charge exchange $\cdot$ Long-lived radionuclide detection · Molecule dissociation

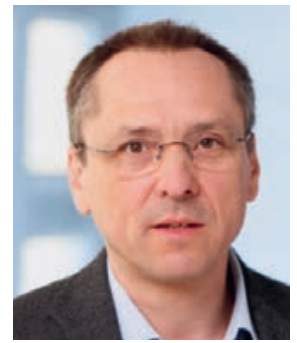

Hans-Arno Synal, accelerator mass spectrometry specialist with a background in nuclear and atomic physics, studied Physics at Rheinische Friedrich-Wilhelms-Universität Bonn, and graduated in 1985 . He moved to Swiss Federal Institute of Technology Zurich (ETHZ) and obtained his $\mathrm{PhD}$ on 'Accelerator mass spectrometry with ${ }^{36} \mathrm{Cl}$ ' in 1989 . Following a post-doctoral fellowship at the Institute of Intermediate Energy, ETH Zurich, he became research scientist at the Paul Scherrer Institute. Since 2008, he is heading the ETH Laboratory of Ion Beam Physics (LIP) and he was appointed as honorary Professor at ETH Zurich in 2011.

\section{Introduction}

Radioactivity is often perceived as a threat because of the enormous energy release during a single nuclear transformation. But it is precisely this fundamental property that ultimately causes it to disappear and according to the half-life of the nuclides, their number decreases exponentially. Thus, only a few surviving radionuclides exist on Earth today, which were created during the stellar explosion of the precursor star of our solar system. In general, detection of radionuclides is relatively easy since radioactive decay is accompanied by high-energy radiation and a single decaying atom can be detected. For long-lived nuclides, however, the decay probability is so low that many nuclides must be present to detect a statistically significant number of decays within a reasonable time. Not only primordial radionuclides are found in nature today. Radionuclides are constantly being produced in connection with the decaying primordial radionuclides $\mathrm{U}$ and $\mathrm{Th}$ as well as from the radiation generated by their decay products. In addition, they are produced by cosmic radiation, and artificially produced nuclides from the worldwide military and civil nuclear activities are found in the environment. Their well-defined source mechanism is a great advantage. It enables us to use them as tracer substances. Natural production rates are usually very low, and these nuclides occur in extremely low isotope ratios compared to their stable isotopes. Typically, they are within a range of $10^{-9}$ to
$10^{-15}$ or even below. This makes detection using conventional mass spectrometers practically impossible.

In the middle of the last century, when the properties of ${ }^{14} \mathrm{C}$ were studied, attention was also focused on its decay properties when it was discovered that the activity of dead organic material was an exceptional measure for dating its origin. ${ }^{[1]}$ It was clear early on that with a half-life of 5700 years, direct mass spectrometric detection of individual atoms is many times more efficient than patiently waiting for individual decay events. ${ }^{[2]}$ But the almost infinite surplus of stable carbon isotopes and the presence of practically equal-mass nuclear and molecular isobars put paid to the dream of directly counting the ${ }^{14} \mathrm{C}$ atoms. Only when it was realized that the nuclear ${ }^{14} \mathrm{C}$ isobar ${ }^{14} \mathrm{~N}$ could be completely suppressed by creating negatively charged ions ${ }^{[3]}$ and with the knowledge that interfering mass-equivalent molecules can be completely broken up into atomic ions by the charge changing process in the stripper of a tandem accelerator ${ }^{[4]}$ the possibility for mass spectrometric ${ }^{14} \mathrm{C}$ detection appeared. ${ }^{[5]}$ This discovery was the birth of accelerator mass spectrometry (AMS), which spread rapidly as the huge potential and enormous advantages of using direct ${ }^{14} \mathrm{C}$ detection were immediately recognized. Today, there is a wide variety of radionuclides which are routinely analysed with AMS systems. ${ }^{[6]}$ Radiocarbon is still the most important and, based on its unique biochemical behavior, has countless applications. ${ }^{10} \mathrm{Be},{ }^{26} \mathrm{Al},{ }^{36} \mathrm{Cl},{ }^{41} \mathrm{Ca},{ }^{129} \mathrm{I},{ }^{236} \mathrm{U}$, and nuclides of the actinide series can be regarded as standard AMS nuclides with versatile applications in earth/environmental sciences as well in bio-medical application, nuclear forensic, and many other research fields. In addition, there are more exotic nuclides such as ${ }^{32} \mathrm{Si},{ }^{53} \mathrm{Mn},{ }^{60} \mathrm{Fe}$, ${ }^{63} \mathrm{Ni}$ and others that have been measured using AMS technology in various applications. The focus of this article is the basic technology which makes the detection of such nuclides at their natural abundance level possible.

\section{Characteristics of AMS Systems}

A typical AMS setup as shown in Fig. 1 has the following main components: i) ion source, ii) pre-acceleration mass filtering system, iii) acceleration stage with charge exchange and molecule dissociation unit, iv) post acceleration mass filtering 